\title{
CONTENIDO DE BORO EN PLANTACIONES DE CAFÉ (COFFEA ARABICA L.) EN DOS LOCALIDADES CAFETALERAS DE VENEZUELA
}

\author{
BORON CONTENT IN COFFEE PLANTATIONS (COFFEA ARABICA L.) \\ IN TWO COFFEE PRODUCING LOCATIONS OF VENEZUELA
}

\author{
Auristela Malavé ${ }^{1}$ Pablo Carrero ${ }^{2}$; Mairin Lemus $^{3}$; Mariana García ${ }^{4}$
}

\begin{abstract}
RESUMEN
Con el objetivo de determinar el estatus de boro en plantaciones de café (Coffea arabica L.) para Venezuela se muestrearon dos localidades cafetaleras venezolanas. Con veinte unidades experimentales por área se tomaron muestras de plantas de café y sus respectivos suelos en las localidades de Santa Cruz de Mora (Estado Mérida) y Caripe (Estado Monagas), durante la época de cosecha para determinar el contenido de boro sin el suministro de algún aditivo contentivo del elemento en estudio. Las muestras de suelo y tejido de planta se trataron mediante un procedimiento de extracción en caliente con ácido nítrico previo a la determinación espectrofotométrica de boro con azometina-H usando un sistema de flujo continuo. El tratamiento estadístico de los datos obtenidos para el contenido del elemento en las muestras de frutos y hojas fueron 24,96 $\pm 2,39 ; 75,86 \pm 2,79 \mu \mathrm{g} \mathrm{g}^{-1}$ (Santa Cruz de Mora) y $9,56 \pm 1,12 ; 29,08 \pm 2,15 \mu \mathrm{g} \mathrm{g}^{-1}$ (Caripe) masa, seca respectivamente, los cuales muestran diferencias significativas en el contenido de boro tanto entre localidades como entre tejidos $(p<0,001)$, mientras que en suelos fueron $9,08 \pm 1,38 \mu \mathrm{g} \mathrm{g}{ }^{-1}$ (Santa Cruz de Mora) y 4,42 $\pm 0,95 \mu \mathrm{g} \mathrm{g}^{-1}$ masa seca, los cuales muestran diferencias significativas entre localidades $(p<0,001)$. Estos resultados indicaron valores de referencia para el contenido del elemento mayores en Santa Cruz de Mora (Estado Mérida) que en Caripe (Estado Monagas).
\end{abstract}

Palabras clave: Coffea arabica, suelo, contenido de boro, azometina-H.

\begin{abstract}
With the object of determining the boron status in coffee plantations for Venezuela, two Venezuelan coffee producing locations were sampled. With twenty Experimental units per area, samples of coffee plants and their respective soils were taken from Santa Cruz de Mora (Mérida State) and Caripe (Monagas State), during harvest to determine the boron content without any additive with the element in study. The soil and tissue plant samples were treated through a hot extraction procedure prior to spectrophotometric determination of boron with azomethine- $H$ using a continuous flow system. The statistical analysis of results for the element content in fruit and leaf samples were 24.96 $\pm 2.39,75.86 \pm 2.79 \mu \mathrm{g} \mathrm{g}^{-1}$ (Santa Cruz de Mora) and 9.56 $\pm 1.12,29.08 \pm 2.15 \mu \mathrm{g} \mathrm{g} \mathrm{g}^{-1}$ (Caripe) dry weight respectively, which show significative differences in the boron content between areas and tissues $(p<0,001):$ while for the soils were $9.08 \pm 1.38 \mu \mathrm{g} \mathrm{g}^{-1}$ (Santa Cruz de Mora) and $4.42 \pm 0.95 \mu \mathrm{g} \mathrm{g}^{-1}$ (Caripe) dry weight, which show significative differences in the boron content inter areas $(p<0,001)$. These results indicated higher reference values for the element content in Santa Cruz (Mérida State) than in Caripe (Monagas State).
\end{abstract}

Key words: Coffea arabica, soil, boron content, azomethine- $H$.

\footnotetext{
1 Núcleo de Monagas-Universidad de Oriente, Venezuela.

2 Facultad de Ciencias-Universidad de los Andes, Venezuela.

3 Núcleo de Sucre-Universidad de Oriente, Venezuela.

4 INIA Monagas-Estación Experimental local Caripe, Estado Monagas, Venezuela. e-mail: auris@ula.ve
} 


\section{INTRODUCCIÓN}

El boro (B) es un micronutrimento esencial requerido por las plantas para su normal desarrollo y crecimiento. Sin embargo, a través de la historia de investigación de este elemento, ha sido establecido que los rangos de concentración en la solución del suelo que causan síntomas de deficiencia o toxicidad en las plantas son más pequeños que para cualquier otro elemento, aunado a la situación de que dichos rangos varían de acuerdo con la especie; es decir, un intervalo de concentraciones de B puede ser normal para un determinado tipo de plantas mientras que para otras puede resultar tóxico o deficiente, lo que ha tenido una profunda influencia para un mejor conocimiento del comportamiento del B en los suelos, estando su disponibilidad afectada por diversos factores tales como $\mathrm{pH}$, textura, humedad, contenido y calidad de materia orgánica y tipo y contenido de arcillas (Malavé, 2005). La carencia de este micronutrimento en las plantas causa problemas tales como la muerte del centro de crecimiento, el desarrollo lento de las raíces y otros síntomas dependiendo de la severidad de la deficiencia (Camacho-Cristóbal et al., 2002, 2005; Molassiotis et al., 2005; Warncke, 2005), mientras que su exceso es tóxico (Lægreid et al., 1999; Ruiz et al., 2003; Apostol, et al., 2004; Papadakis et al., 2004a; 2004b; Reid, et al., 2004; Ghanati et al., 2005). Recientemente se ha indicado que la base de la esencialidad del B radica en la estabilización de moléculas con grupos diol-cis volviéndolos efectivos indistintamente de su función en las plantas (Bolaños et al., 2004), donde se ha sugerido su participación en los procesos de mantenimiento de la estructura de la pared celular, mantenimiento de la función de la membrana y de soporte en actividades metabólicas como funciones del crecimiento y desarrollo. En base a lo anterior, existen evidencias circunstanciales que sugieren con fuerza que el $\mathrm{B}$ probablemente es un micronutrimento esencial para animales superiores y humanos (Nielsen, 2000; Hunt, 2003; Devirian y Volpe, 2003; Barranco y Eckert, 2004; Park et al. 2004; Ralston y Hunt, 2004), sin obviar que su consumo en exceso puede resultar tóxico (ATSDR, 2007; Malina, 2004), con una dosis de consumo del elemento sugerida para adultos de hasta $13 \mathrm{mg} /$ día (WHO/FAO/IAEA, 1996) a partir de alimentos de origen vegetal que constituyen su principal fuente de consumo (Gupta y Gupta, 2005).
El presente trabajo tiene como finalidad determinar el contenido de boro en plantaciones de café de dos áreas cafetaleras de Venezuela, ubicadas en el Estado Mérida (Santa Cruz de Mora) y en el Estado Monagas (Caripe), para así tener un estatus de valores de referencia en cuanto al contenido de este elemento tanto en plantas como en suelos para ambas localidades.

\section{MATERIALES Y MÉTODOS}

Las muestras de suelos y plantas analizadas en el presente trabajo corresponden a plantaciones de café (Coffea arabica), provenientes de dos localidades cafetaleras distintas de Venezuela: Santa Cruz de Mora (Estado Mérida) y Caripe (Estado Monagas) (Fig. 1). Santa Cruz de Mora es una importante localidad productora y distribuidora de café, ubicada dentro del valle del Río Mocotíes en los Andes Venezolanos, a $622 \mathrm{msnm}\left(71^{\circ} 32^{\prime}-71^{\circ} 43^{\prime}\right.$ longitud oeste, $08^{\circ} 16^{\prime}-08^{\circ} 33^{\prime}$ latitud norte), con clima seco subhúmedo, precipitación anual alrededor de los $900 \mathrm{~mm}$ y temperatura media de $24{ }^{\circ} \mathrm{C}$, donde abundan suelos del orden inceptisol (Zerpa, 2006). Caripe es un importante centro turístico ubicado en el macizo montañoso del turimiquire en Venezuela, a $1.120 \mathrm{msnm}\left(63^{\circ} 11^{\prime}-63^{\circ} 37^{\prime}\right.$ longitud oeste, $10^{\circ}$ $4^{\prime}-10^{\circ} 19^{\prime}$ latitud norte), caracterizado por un clima de montaña, precipitación promedio anual de 1.124 mm y temperatura entre 12 y $24^{\circ} \mathrm{C}$ con vegetación de bosque húmedo premontano, cuyos suelos son mayormente del orden inceptisol (Atlas del Estado Monagas, 2000).

\section{TOMA Y CONSERVACIÓN DE MUESTRAS}

En base a un diseño de veinte unidades experimentales por localidad correspondientes a tres muestras (suelo, hojas y frutos) para cada área de estudio, se colectó un total de 60 muestras tanto para Santa Cruz de Mora como para Caripe. Las muestras de tejido de plantas y suelos de Santa Cruz de Mora se colectaron en septiembre del 2004 y las de Caripe en enero del 2005, debido a la diferencia en las épocas de cosecha para cada localidad.

Las muestras tanto de frutos como de hojas se colectaron directamente de cada planta tomando las hojas del tercio medio y el cuarto par desde el ápice de cada planta. Ambos tipos de muestras se 


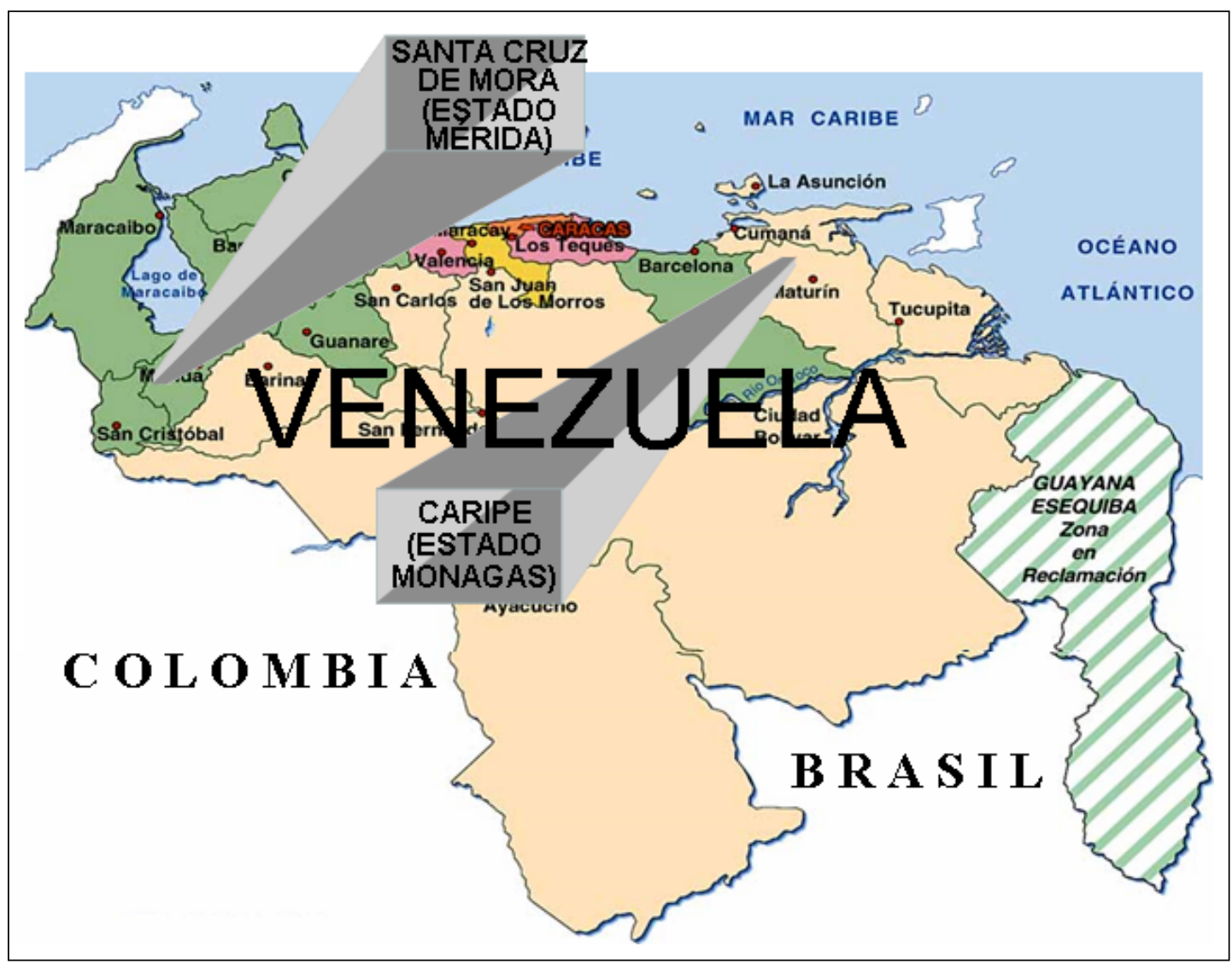

Figura 1. Ubicación geográfica de las dos localidades cafetaleras venezolanas en estudio: Santa Cruz de Mora (Estado Mérida) y Caripe (Estado Monagas).

secaron en un horno a $70^{\circ} \mathrm{C}$ durante tres días, luego pulverizadas con un molino analítico y guardadas en un desecador hasta el pretratamiento de las mismas para el análisis.

Las muestras de suelos se colectaron directamente del pie de las plantas en la proyección de la copa del árbol. Luego se trasladaron al laboratorio, se secaron al aire durante cinco días y, después de la remoción de las piedras y restos vegetales, posteriormente cada una fue macerada, tamizada con un tamiz de 80 mesh y finalmente guardadas en un desecador hasta el pretratamiento de las mismas para el análisis.

Todas las soluciones se prepararon con agua destilada y desionizada $18 \mathrm{M} \Omega \mathrm{cm}^{-2}$ en envases de polimetilpenteno, los cuales, además del resto del material utilizado, se mantuvieron en remojo durante 24 horas en una solución de $\mathrm{HNO}_{3}$ al $10 \%$ y finalmente lavados varias veces con agua desionizada.

\section{PRETRATAMIENTO DE LAS MUESTRAS}

Todas las muestras se trataron por un procedimiento de extracción de boro en caliente usando $\mathrm{HNO}_{3} 1,0 \mathrm{M}$ de acuerdo a Al-Ammar et al. (2000). En cada caso se tomaron alícuotas de 2,0 g de cada muestra (frutos, hojas y suelos), las cuales se colocaron en un recipiente con $15 \mathrm{~mL}$ de $\mathrm{HNO}_{3} 1,0$ mol L ${ }^{-1}$; después se taparon y se colocaron en un horno a $80^{\circ} \mathrm{C}$ durante una hora. Luego se enfriaron hasta temperatura ambiente, se filtraron y evaporaron a sequedad mediante calentamiento suave en una plancha y se diluyeron (con ayuda de un baño ultrasónico) con ácido sulfúrico concentrado hasta un volumen de $25 \mathrm{~mL}$.

\section{ANÁLISIS DE LAS MUESTRAS}

El análisis de las diferentes muestras se realizó utilizando el método de flujo continuo recien- 
temente descrito por Carrero et al. (2005) para determinación de boro mediante generación de metil borato con detección espectrofotométrica usando azometina-H. Cada muestra, previamente pretratada en $\mathrm{H}_{2} \mathrm{SO}_{4}$ concentrado, es combinada con metanol en una proporción 1:3 (v/v) para que la reacción de esterificación sea suficientemente exotérmica, produciendo el calor necesario para generar el metil borato gaseoso sin fuente de calentamiento externa. Subsecuentemente, este producto es separado de la mezcla de reacción en un separador líquido/gas usando $\mathrm{N}_{2}$ como gas de arrastre y luego se hidroliza el ácido bórico con una solución buffer $\left(\mathrm{NH}_{4}{ }^{+} /\right.$ $\left.\mathrm{PO}_{4}{ }^{-3}, \mathrm{pH}=6,8\right)$. Finalmente la nueva mezcla es separada en un segundo separador líquido/gas y la fase líquida es combinada con azometina-H para formar el complejo de boro cuya absorbancia es medida a $420 \mathrm{~nm}$.

Para las medidas de la absorbancia se utilizó un espectrofotómetro Varian 634 equipado con una celda de cuarzo para flujo continuo (Starna cells, Inc., USA) de $3 \mathrm{~mm}$ de diámetro interno, $10 \mathrm{~mm}$ de longitud y $70 \mu \mathrm{L}$ de capacidad. Como sistemas de propulsión se utilizaron dos bombas peristálticas Gilson Minipuls de cuatro canales ambas equipadas con un sistema que permite variar la velocidad de flujo. Además, se utilizaron un ultrasonido (Branson 2210), un molino analítico, un tamiz de 80 mesh, un horno de secado y una plancha de calentamiento.

Para el análisis estadístico descriptivo simple de los datos se utilizó el paquete "SPSS versión 7.5" (2002), en la determinación de las medias del contenido del elemento, con su respectiva desviación estándar, y para la comparación de las mismas tanto entre tejidos como entre localidades.

\section{RESULTADOS Y DISCUSIÓN}

Como ya se sabe, el desarrollo y crecimiento óptimo de las plantas se logra cuando las condiciones del suelo son adecuadas para proporcionar todos los elementos esenciales necesarios incluyendo al boro, por lo que es necesario determinar su estatus en muestras agrícolas a manera de recabar la mayor cantidad de información para predecir si las cantidades de boro disponible son adecuadas (Malavé, 2005).

Los valores promedios del contenido de boro correspondientes a los tejidos de plantas se presentan en la tabla 1, donde se puede observar que las plantas eficientemente concentran la mayor cantidad del elemento en sus hojas, encontrándose las mayores concentraciones en la localidad de Santa Cruz de Mora (Estado Mérida). Así, el contenido del elemento para los tejidos de plantas de la región merideña es al menos el doble con respecto a las muestras de la región monaguense. Adicionalmente, en base al tratamiento estadístico de los datos, se encontró que existen diferencias significativas en el contenido de boro tanto entre localidades como entre tejidos $(p<0,001)$, estando estos valores dentro del rango de concentración de hojas, frutos y semillas comestibles que va de 5 a $85 \mu \mathrm{g} \mathrm{g}^{-1}$ de muestra seca (Arruda y Zagatto, 1987; Tsalev y Zaprianov, 1983).

Los valores promedio correspondientes al contenido de boro para los suelos de ambas regiones están representados en la fig. 2, los cuales

\section{Tabla 1}

Contenido de boro ( $\mu \mathrm{g} \mathrm{g}^{-1}$ de masa seca) en las muestras de tejidos de plantas de las áreas de Santa Cruz de Mora (Estado Mérida) y Caripe (Estado Monagas)

\begin{tabular}{|l|l|c|}
\hline \multicolumn{1}{|c|}{ Localidad } & Tejidos & media \pm SD \\
\hline Santa Cruz & Frutos & $24,96 \pm 2,39$ \\
de Mora & Hojas & $75,86 \pm 2,79$ \\
(Estado Mérida) & & \\
\hline Caripe & Frutos & $9,56 \pm 1,12$ \\
(Estado Monagas) & Hojas & $29,08 \pm 2,15$ \\
\hline
\end{tabular}

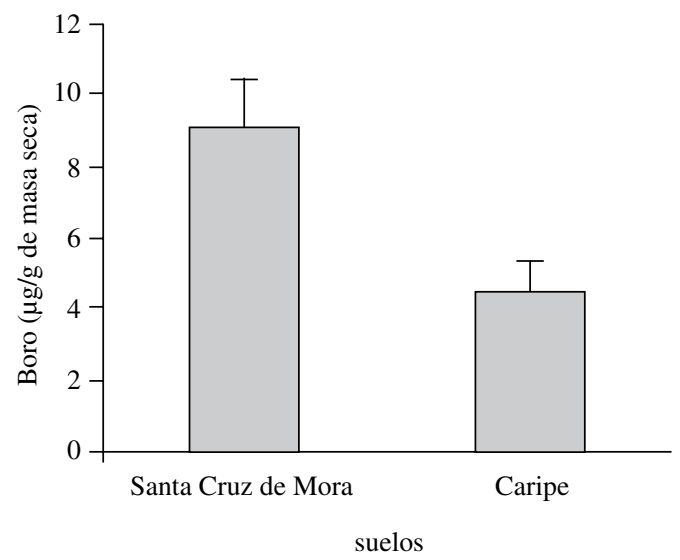

Figura 2. Contenido de boro ( $\mu \mathrm{g} \mathrm{g}^{-1}$ de masa seca) en las muestras de suelos de las áreas en estudio: Santa Cruz de Mora (Estado Mérida) y Caripe (Estado Monagas). 
muestran diferencias significativas en el contenido del elemento entre localidades $(p<0,001)$. En este caso igualmente se destaca el mayor contenido del elemento para las muestras de suelo de la región merideña, lo cual está en concordancia con los resultados obtenidos para las muestras de tejidos de plantas. No obstante, los resultados obtenidos para el contenido de boro en las muestras de suelos para ambas localidades están dentro de los valores normales de los suelos con bajo contenido de boro $\left(<10 \mu \mathrm{g} \mathrm{g}^{-1}\right)$ que componen a la mayoría de los suelos del país, mientras que los de alto contenido (10-100 $\left.\mu \mathrm{g} \mathrm{g}^{-1}\right)$ corresponden a suelos próximos a sitios con actividad volcánica y geológica (Power y Woods, 1997), no siendo este último el caso de las áreas aquí estudiadas.

A pesar de que ambas localidades experimentales son valles con características edafoclimáticas bastante similares, los resultados son concluyentes en cuanto a los diferentes contenidos de boro en los suelos, lo que igualmente resulta en la diferencia para su contenido en los tejidos de plantas (hojas y frutos) para cada sitio en estudio. Sin embargo, es necesario destacar la ubicación de Santa Cruz de Mora dentro del Valle de Mocotíes cuya altura

\section{LITERATURA CITADA}

AL-AMMAR, A., REITZNEROVÁ, E. Y BARNES R.M. 2000. Determination of boron in biological samples. J. Radianal. Nucl. Chem. 244(2): 267-272.

APOSTOL, K. Y J. ZWIAZEK. 2004. Boron and water uptake in jack pine (Pinus banksiana) seedlings. Environ. Exp. Bot., 51(2): 145-153.

ARRUDA, M. Y ZAGATTO E. 1987. A simple stop-flow method with continuous pumping for the spectrophotometric flow-injection determination of boron in plants. Anal. Chim. Acta. 199(1): 137-145.

ATLAS DEL ESTADO MONAGAS. 2000. Ministerio del Ambiente y Recursos Naturales Renovables, Venezuela, pp. 78-79.

ATSDR (AGENCY FOR TOXIC SUBSTANCES AND DISEASE REGISTRY). 2007. Draft Toxicological Profile for Boron. http://www.atsdr.cdc.gov/toxprofiles/tp26.pdf (Última visita 22 de noviembre de 2007).

BARRANCO, W. Y D. ECKHERT. 2004. Boric acid inhibits human prostate cancer cell proliferation. Cancer Lett., 216(1): 21-29.

BOLAÑOS, L.; LUKASZEWSKI, K.; BONILLA, I. Y BLEVINS, D. 2004. Why boron? Plant Physiol. Biochem. 42(11): 907-912.

CAMACHO-CRISTÓBAL, J.; ANZELLOTTI, D. Y GONZÁLEZ-FONTES, A. 2002. Changes in phenolic metabolism of tobacco plants during short-term boron deficiency. J. Plant Physiol., 40(12): 997-1002. global oscila entre 520 y $3.249 \mathrm{msnm}$, estando esta localidad insertada en las zonas más bajas a $622 \mathrm{msnm}$ (Zerpa, 2006), quedando prácticamente impedida de la pérdida de nutrimentos por erosión hídrica, situación que sí se presenta en la localidad de Caripe en forma moderada debido a sus pendientes (Tenias et al., 1992), lo que podría explicar a grandes rasgos esta diferencia para el contenido de boro entre ambas localidades experimentales. Finalmente, se puede destacar la obtención de valores de referencia, no encontrados en la literatura para Venezuela, en cuanto a la concentración del elemento en plantaciones de café para la zona del Estado Mérida mayores que para la zona del Estado Monagas, lo que alerta a los productores de esta última zona a continuar un monitoreo de los niveles de boro en los suelos antes de proceder a realizar plantaciones.

\section{AGRADECIMIENTO}

Los autores agradecen al Consejo de Investigación de la Universidad de Oriente, Venezuela, por el apoyo brindado a la presente investigación.
CAMACHO-CRISTÓBAL, J.; MALDONADO, J. Y GONZÁLEZ-FONTES, A. 2005. Boron deficiency increases putrescine levels in tobacco plants. J. Plant Physiol., 162(8): 921-928.

CARRERO, P.; MALAVÉ, A. Y ROJAS, E. 2005. On-line generation and hydrolysis of methyl borate for the spectrophotometric determination of boron in soil and plants with azomethine-H, Talanta, 68(2): 374-371.

DEVIRIAN, T. Y VOLPE, S.L. 2003. The physiological effects of dietary boron. Crit. Rev. Food Sci. Nutr. 43(2): 219-231.

GHANATI, F.; MORITA, A. Y YOKOTA, H. 2005. Deposition of suberin in roots of soybean induced by excess boron. Plant Sci., 168(2): 397-405.

GUPTA, U. Y GUPTA, S. 2005. Future trends and requirements in micronutrient research. Comm. Soil Sci. Plant Anal., 36(1-3): 33-45.

HUNT, C.D. 2003. Dietary boron: an overview of the evidence for its role in immune function. J. Trace Elem. Exp. Med. 16(4): 291-306.

L/EGREID, M.; BØCKMAN, O.C. Y KAARSTAD, O. 1999. Agriculture, fertilizers, and the environment, CABI Publishing in association with Norsk Hydro ASA, New York. 136 p.

MALAVÉ, A. 2005. Los suelos como fuente de boro para las plantas. Rev. Cientif. UDO-Agrícola, 5(1): 10-26.

MALINA, G. 2004. Ecotoxicological and environmental problems associated with the former chemical plant in Tarnowskie Gory, Poland. Toxicol., 205(3): 157-172. 
MOLASSIOTIS, A.; SOTIROPOULOS, T.; TANOU, G.; DIAMANTIDIS G. y THERIOS, I. 2006. Boron-induced oxidative damage and antioxidant and nucleolytic responses in shoot tips culture of the apple rootstock EM 9 (Malus domestica Borkh). Environ. Exp. Bot., 56(1): 54-62.

NIELSEN, F. 2000. Trace elements in human health and disease: an update importance of making dietary recommendations for elements designated as nutritionally beneficial, pharmacologically beneficial, or conditionally essential. J. Trace Elem. Exp. Med. 13(1): 113-129.

PAPADAKIS, I.; DIMASSI, K.N.; BOSABALIDIS, A.; THERIOS, I.; PATAKAS, A. Y GIANNAKOULA, A. 2004a. Boron toxicity in 'clementine' mandarin plants grafted on two rootstocks. Plants Sci., 166(2): 539-547.

PAPADAKIS, I.; DIMASSI, K.N.; BOSABALIDIS, A.; THERIOS, I.; PATAKAS, A. Y GIANNAKOULA, A. 2004b. Effects of B excess on some physiological parameters of 'navelina' orange plants grafted on two rootstocks. Environ. Exp. Bot., 51(3): 247-257.

PARK, M.; LI, Q.; SHCHEYNIKOV, N.; ZENG, W. Y MUALLEM, S. 2004. NaBC1 is a ubiquitous electrogenic $\mathrm{Na}^{+}$-coupled borate transporter essential for cellular boron homeostasis and cell growth and proliferation. Molec. Cell, 16(3): 331-341.

POWER, P. Y WOODS, W. 1997. The chemistry of boron and its speciation in plants. Plant Soil. 193(1-2): 1-13.
RALSTON, N. Y HUNT, C. 2004. Transmembrane partitioning of boron and other elements in RAW 264.7 and HL60 cell cultures. Biol. Trace Elem. Res. 98(2): 181-192.

REID, R.; HAYES, J.; POST, A.; STANGOULIS, J. Y GRAHAM, R. 2004. A critical analysis of the cause of boron toxicity in plants. Plant Cell Environ., 27(11): 1405-1414.

RUIZ, J.; ROSA, R.M. Y ROMERO, L. 2003. Preliminary studies on the involvement of biosynthesis of cysteine and glutathione concentration in the resistance to B toxicity in sunflower plants. Plant Sci., 165(4): 811-817.

SPSS. REAL STATISTIC. 2002. Version 7.5 English and Spanish. Serial: 4117631.

TENIAS, J.; BLANCO, F.; VELÁSQUEZ, E.; SÁNCHEZ, A. Y ARIAS, L. 1992. Diagnóstico Agroecológico de la región Nor-oriental. Centro de Investigaciones Agropecuarias de la Región Nor-oriental (CIARNO). Maturín, Venezuela, 36 p.

TSALEV, D. Y ZAPRIANOV, Z. 1983. Atomic absorption spectrometry in occupational and health practice. Vol 1, Florida. 103 p.

WARNCKE, D. 2005. Ameliorating internal black spot in cranberry bean seed with boron application. Comm. Soil Sci. Plant Anal., 36(4-6): 775-781.

WHO/FAO/IAEA. 1996. Trace elements in human nutrition and health. World Health Organization. Geneva. pp. 175-179.

ZERPA, M. 2006. Caracterización geográfica Santa Cruz de Mora. http://www.monografias.com/trabajos36/santa-cruz-mora/santacruz-mora.shtml (Última visita 18 de agosto de 2006). 